\title{
What can we learn from fMRI capture of visual hallucinations in Parkinson's
} disease?

Kathy Dujardin ${ }^{1,3}$, David Roman ${ }^{4}$, Guillaume Baille ${ }^{1,3}$, Delphine Pins ${ }^{2}$, Stéphanie Lefebvre2, Christine Delmaire ${ }^{5}$, Luc Defebvre ${ }^{1,3}$, Renaud Jardri ${ }^{2,4}$

${ }^{1}$ Univ. Lille, Inserm, U1171 - Degenerative \& vascular cognitive disorders, F-59000 Lille, France

${ }^{2}$ Univ. Lille, CNRS, UMR 9193 - Cognitive and Affective Sciences - SCALab, F-59000 Lille, France

${ }^{3} \mathrm{CHU}$ Lille, Neurology and Movement Disorders Department, F-59000 Lille, France

${ }^{4} \mathrm{CHU}$ Lille, Psychiatry Department, F-59000 Lille, France

${ }^{5} \mathrm{CHU}$ Lille, Neuroimaging Department, F-59000 Lille, France

*Corresponding author:

Kathy Dujardin, Neurologie et Pathologie du Mouvement, Neurologie A, Hôpital Salengro, Centre Hospitalier Universitaire, F-59037 Lille cedex, France

KEYWORDS: hallucination capture / two-step method / default-mode network / connectivity RUNNING TITLE: Chronic visual hallucinations in PD

MANUSCRIPT WORD COUNT: 2010

ABSTRACT WORD COUNT: 232

Title Character count: 84

30 references, 1 figure, 1 table 


\section{Abstract}

Background: With disease progression, patients with Parkinson's disease (PD) may have chronic visual hallucinations (VH). The mechanisms behind this invalidating non-motor symptom remain largely unknown, namely because it is extremely difficult to capture hallucination events. This study aimed to describe the patterns of brain functional changes when $\mathrm{VH}$ occur in PD patients.

Methods: Nine PD patients were enrolled because of their frequent and chronic VH (> 10/day). Patients with severe cognitive decline (MMSE<18) were excluded. Patients were scanned during ON/OFF hallucinatory states and resting-state functional imaging ( $r$ s-fMRI) was performed. Data were analyzed in reference to the two-step method, which consists in: (i) a data-driven analysis of per-hallucinatory fMRI data, and (ii) selection of the components of interest based on a post-fMRI interview.

Results: The phenomenology of $\mathrm{VH}$ ranged from visual spots to distorting faces. First, at the individual level, several $\mathrm{VH}$-related components of interest were identified and integrated in a second-level analysis. Using a random-effects self-organizing-group ICA, we evidenced increased connectivity in visual networks concomitant to $\mathrm{VH}$, encompassing $\mathrm{V} 2, \mathrm{~V} 3$ and the fusiform gyri bilaterally. Interestingly, the stability of the default-mode network (DMN) was found positively correlated with VH severity (spearman's rho $=0.77, p=0.05$ ).

Conclusion: By using a method that does not need online self-report, we showed that $\mathrm{VH}$ in PD patients were associated with functional changes in associative visual cortices, possibly linked with strengthened stability of resting-state networks. 
Parkinson's disease (PD) is a neurodegenerative disease resulting from progressive death of the dopaminergic neurons in the brainstem, particularly the pars compacta of the substantia nigra. Besides the hallmark motor symptoms (rest tremor, hypokinesia, rigidity and postural instability), PD patients have non-motor symptoms. Neuropsychiatric disorders are among the most frequently reported non-motor symptoms, occurring in up to $60 \%$ of the patients [Martinez-Martin et al., 2007]. Depression, anxiety, apathy and psychosis are the most common. Visual hallucinations (VH) are the most typical psychotic symptom [Friedman, 2010]. Symptoms of VH usually form a continuum progressing with the course of the disease [Ffytche and Aarsland, 2017]. Passage (feeling like something is passing at the outer visual field) and illusions (mis- or distorted perception of an actual stimulus) are the most common symptoms at early stage of PD. As the disease progresses, VH may be complex and formed, usually vision of animals, people or objects. The scene is often stereotyped. The hallucinations usually ignore the patient and disappear when approaching. They tend to occur in a low sensory environment. Initially, insight is preserved. Later, it can be lost and contribute to the development of delusions. Such experiences whose frequency ranges from 22 to $37 \%$ [Fenelon and Alves, 2010], are often more chronic and cause more distress to patients and their caregivers.

The mechanisms behind VH remain largely unknown. Since these symptoms may be induced by medication (namely, dopamine agonists), dopaminergic pathways were presumably involved, but not only [Fénelon et al., 2000] [Friedman, 2010]. A number of studies have shown the benefit of acetylcholinesterase inhibitors [Burn et al., 2006] [Swann and O'Brien, 2018], suggesting involvement of the cortical cholinergic pathway. Moreover, visual dysfunction is frequent in patients with PD. Reduction in contrast sensitivity and color discrimination due to degeneration of retinal dopamine receptors [Pieri et al., 2000] [Diederich et al., 2009] have been described. A thinning of the retinal nerve fiber layer was reported by optical coherence tomography in PD patients with visual hallucinations [Lee et al., 2014]. Nevertheless, the contribution of these retinal phenomena to VH remains open to discussion [Weil et al., 2016]. The structural and functional brain imaging studies showed changes in visual and more high-functioning networks in PD patients suffering from $\mathrm{VH}$ [Diederich et al., 2009; Shine et al., 2014]. By comparing the patterns of brain activation in patients with and without chronic $\mathrm{VH}$, some authors notably showed decreased activations in both dorsal and ventral visual pathways in hallucinated patients [Lenka et al., 2015]. Despite these important trait-imaging findings, a better understanding of the pathophysiology of chronic $\mathrm{VH}$ in PD lacks a direct exploration of the brain changes associated with $\mathrm{VH}$ occurrences (i.e., state or capture studies).

Because of the challenging nature of state explorations of hallucinations, only one single-case study reported the results of a functional MRI (fMRI) scan performed in a 66-year old PD patient with severe $\mathrm{VH}$ and who signaled their occurrence by pressing a button while scanning [Goetz et al., 2014]. By comparing epochs with and without VH, these authors observed an increased activation in bilateral cingulate gyri, and, in the right medial frontal, and postcentral gyri, as well as the right thalamus and brainstem. Decreased activations were also reported in the right primary visual cortex. The authors interpreted these patterns as 


\section{Methods}

\section{Participants}

Nine PD patients with chronic VH participated in the study between March 2014 and August 2017. All met the UK Brain Bank criteria for idiopathic PD [Gibb and Lees, 1988] and none was suffering from a neurological disease other than PD. They were outpatients of the movement disorders clinic at the university medical center of Lille (CHU Lille, France). They were invited to participate in the study if they had $\mathrm{VH}$ episodes occurring more than ten times per day. All participants had normal or corrected-to-normal vision.

Patients with multisensory hallucinations, with severe cognitive decline (i.e., who scored below 18 at the Mini Mental State Examination), those presenting a depressive episode, those younger than 40 and older than 80 years or having contra-indications to MRI were excluded likewise. All patients were scanned in the "ON-drug" state, after receiving their usual doses of antiparkinsonian medication. Psychotropic and anti-parkinsonian treatments needed to be stable during at least the last 30 days before inclusion.

All participants gave their informed consent to participation in the study, which had been approved by the local institutional review boards (CPP Nord-Ouest IV, 2013-A00531-44).

\section{Assessments}

The general presence of $\mathrm{VH}$ was detected using the hallucination item of the Neuropsychiatric Inventory-Clinician (NPI-C) [de Medeiros et al., 2010]. VH severity and frequency were then rated with a Visual Analogical Scale and VH intensity with the University of Miami Parkinson's disease Hallucinations Questionnaire (UMPDHQ, [Papapetropoulos et al., 2008]). At inclusion, patients' medications were recorded, and doses of antiparkinsonian medication were converted to levodopa equivalent daily dose (LEDD) [Tomlinson et al., 2010]. The severity of motor symptoms was assessed by the Movement Disorders Society - Unified Parkinson's disease Rating Scale [Goetz et al., 2008] (MDS-UPDRS) (part III), and disease stage by the Hoehn \& Yahr score [Hoehn and Yahr, 1998]. Finally, the severity of depression was quantified using MADRS scores [Montgomery and Asberg, 1979]. Cognitive status was assessed by the scores at the Mini Mental State examination (MMSE) [Folstein et al., 1975] and the Mattis dementia rating scale (MDRS) [Mattis, 1976]. Hand dominance was determined according to the Edinburgh Handedness inventory [Oldfield, 1971].

\section{fMRI acquisition, preprocessing and procedure}


Patients were scanned during ON/OFF hallucinatory states on a 3T MRI scanner (Philips Achieva). High-resolution 3D T1-weighted images were acquired with a magnetizationprepared gradient echo sequence. Resting-state functional imaging (fMRI) was performed with a T2*-weighted EPI sequence lasting $10 \mathrm{~min}$. Patients were required to remain quiet, awake with eyes closed.

Functional data were preprocessed using a slice-scan time correction, a 3D headmotion correction, smoothing with a spatial gaussian filter (FWHM $=6.0 \mathrm{~mm}$ ), a temporal high-pass filtering with $2 \mathrm{sin} / \cos$ and linear trend removal. The anatomical data were subjected to an intensity inhomogeneity correction algorithm, resampled to a $0.5 \mathrm{~mm}^{3}$ resolution, and normalized to the MNI stereotactic space. Data from the head tissue, subcortical structures, and cerebellum were then removed with the aim of advanced cortical segmentation processing. This segmentation was performed at the gray/white matter and the gray matter/cerebrospinal fluid boundaries. A boundary-based registration was finally used to align the functional/anatomical datasets.

We referred to the two-steps fMRI hallucinations capture method, which was previously developed to capture unpredictable events, such as auditory hallucinations in schizophrenia [Jardri et al., 2013; Leroy et al., 2017]. Step 1 is the resting-state fMRI acquisition during ON/OFF hallucinations periods. Step 2 occurs immediately after the MRI acquisition. Using a standardized post-fMRI interview, each participant was asked to report all the sensory experiences that occurred during scanning, including the number of events and their approximate times of occurrence.

\section{Data-analyses}

Data obtained from step 1 were first blindly analyzed using spatial ICA [Formisano et al., 2004]. Among the resulting independent components (IC), the most relevant ones were firstly selected using the IC-fingerprint method [De Martino et al., 2007]. This step allowed us to discard noise-related ICs (e.g., EPI susceptibility, motion artefacts, highfrequency noise), with the aim to only retaining the components associated with a neurophysiological source. Surviving IC were then compared to the post-fMRI interview data, in terms of number of events, times of occurrence, and functional networks of interest (e.g., association visual areas). For an extensive description of the method, its reproducibility and reliability, see [Leroy et al., 2017]. In addition, we generated group IC and their random-effects evaluation using the self-organizing-group approach [Esposito et al., 2005].

Finally, we looked for an interaction between VH and spatial stability of a canonical resting-state network, previously shown associated with hallucinations [Jardri et al., 2013; Lefebvre et al., 2016], i.e., the default-mode network (DMN). To do so, the DMN goodness-offit score (obtained by correlating individual DMN maps with a meta-analytic DMN template) was correlated with $\mathrm{VH}$ severity.

The datasets generated during and/or analyzed during the current study are available from the corresponding author on reasonable request.

\section{Results}

\section{Demographical and clinical characteristics}

Among the nine selected patients, six had and three had not VH episodes during the scan. Their characteristics are presented in Table 1. Data of two patients with VH during the scan had to be discarded due to motion artifacts. At the end, analyses were performed on the data from seven patients (four with and three without VH during the scan). The 
phenomenology of $\mathrm{VH}$ is described in Table 1. It ranged from visual spots to distorting faces (4 episodes per scan and per patient on average).

\section{fMRI analysis}

Referring to the two-step method for hallucinatory-capture, we were able to identify several VH-related components of interest at the individual-level. We notably observed a clear clinical-functional relationship between the phenomenology of $\mathrm{VH}$ and the cortical areas overactivated during hallucinatory experience. This is illustrated in Figure 1-A and $\mathbf{B}$. At the group-level, random-effects self-organizing-group ICA evidenced increased connectivity in visual networks concomitant to $\mathrm{VH}$, encompassing the striate cortex, and the lingual/fusiform gyri, bilaterally (see Figure 1-C). Finally, the spatial stability of the default-mode network (DMN) was found positively correlated with VH severity (Spearman's rho $=0.77, p=0.05-$ Figure 1-D).

\section{Discussion}

By using the two-step method, a recently validated $\mathrm{FMRI}$ procedure that does not need online self-report [Leroy et al., 2017], we showed that VH in PD are associated with functional changes in associative visual cortices. Namely, the occurrence of $\mathrm{VH}$ was concomitant to overactivations of the ventral visual pathways involved in object, animal or face recognition. At the individual level, these per- $\mathrm{VH}$ activations correlated with the phenomenological content of the hallucinatory experiences (e.g., overlap between the per-VH activation map of a patient seeing faces and the fusiform-face-area described in the literature). This suggests that VH in PD corresponds to dysfunctional activations of mental representations, a finding also compatible with the fact that the connectivity between major components of the visual ventral stream that mediates the transformation of visual signal into mental representations was increased in VH [Milner, 2017]. Importantly, the severity of VH was found positively correlated with stability of the DMN, suggesting that $\mathrm{VH}$ in PD could result from increased strength of the DMN, hindering the attentional allocation to the external world. These results are at the opposite of those reported by Goetz et al. when capturing VH in a unique PD patient and contrasting periods with and without VH [Goetz et al., 2014]. Indeed, they observed increased activation in several anterior regions of the brain and decreased activation in the posterior brain regions associated with visual processing. Such discrepancies are difficult to explain at this stage due to the use of different methodological approaches to capture VH: event-related vs. resting-state design, contrast analysis vs. ICA (data-driven approach), one single case vs. group analysis. As the patient had to perform a task in the up-mentioned case report, attention was required. This may explain the increase in activation measured in many frontal regions, the insula and the thalamus.

Our results fit into several current frameworks proposed to account for $\mathrm{VH}$ in PD ([Muller et al., 2014] for a review). Namely, they suggest that endogenous inputs predominate at the expense of exogenous ones, as suggested by the activation, input, modulation model [Diederich et al., 2005]. They also agree with the hypothesis of a disruption of information processing across the attention networks [Shine et al., 2014]. Indeed, the strengthened DMN spatial stability in PD patients with chronic VH could reflect the inability to switch from restingstate activity to oriented attention toward a novel external stimulus. This agrees with Shine et al. [Shine et al., 2015] who observed an increased engagement of the DMN with the primary visual system when eliciting visual misperceptions in PD patients. 
In conclusion, this is the first study capturing functional brain activity concomitant to $\mathrm{VH}$ occurrence in a sample of PD patients. Our results suggest an imbalance between internally-generated and externally perceived information at the roots of VH in PD.

\section{Compliance with Ethical Standards}

- Funding: This study was funded by the Structure Fédérative de Recherche Démence des Maladies Neurologiques et Mentales (DN2M), Lille, France, the European Regional Development Fund (FEDER-Région Nord-Pas-de-Calais, France) and the France Parkinson charity. The sponsors were not involved in the study design or in data interpretation, writing of the report or decision to submit the article for publication.

- Conflict of Interest: The authors have no conflict of interest to declare

- Ethical approval: All procedures performed in studies involving human participants were in accordance with the ethical standards of the institutional and/or national research committee and with the 1964 Helsinki declaration and its later amendments or comparable ethical standards.

- Informed consent: Informed consent was obtained from all individual participants included in the study.

\section{References}


Burn D, Emre M, McKeith I, De Deyn PP, Aarsland D, Hsu C, Lane R (2006): Effects of rivastigmine in patients with and without visual hallucinations in dementia associated with Parkinson's disease. Mov Disord 21:1899-1907.

De Martino F, Gentile F, Esposito F, Balsi M, Di Salle F, Goebel R, Formisano E (2007): Classification of $\mathrm{FMRI}$ independent components using IC-fingerprints and support vector machine classifiers. Neuroimage 34:177-194.

de Medeiros K, Robert P, Gauthier S, Stella F, Politis A, Leoutsakos J, Taragano F, Kremer J, Brugnolo A, Porsteinsson AP, Geda YE, Brodaty H, Gazdag G, Cummings J, Lyketsos C (2010): The Neuropsychiatric Inventory-Clinician rating scale (NPI-C): reliability and validity of a revised assessment of neuropsychiatric symptoms in dementia. Int Psychogeriatr 22:984-994.

Diederich NJ, Fenelon G, Stebbins G, Goetz CG (2009): Hallucinations in Parkinson disease. Nat Rev Neurol 5:331-342.

Diederich NJ, Goetz CG, Stebbins GT (2005): Repeated visual hallucinations in Parkinson's disease as disturbed external/internal perceptions: focused review and a new integrative model. Mov Disord 20:130-140.

Esposito F, Scarabino T, Hyvarinen A, Himberg J, Formisano E, Comani S, Tedeschi G, Goebel $R$, Seifritz E, Di Salle F (2005): Independent component analysis of fMRI group studies by self-organizing clustering. Neuroimage 25:193-205.

Fenelon G, Alves G (2010): Epidemiology of psychosis in Parkinson's disease. J Neurol Sci 289:12-17.

Fénelon G, Mahieux F, Huon R, Ziégler M (2000): Hallucinations in Parkinson's disease: prevalence, phenomenology and risk factors. Brain 123 ( Pt 4):733-745.

Ffytche DH, Aarsland D (2017): Psychosis in Parkinson's Disease. Int Rev Neurobiol 133:585622.

Folstein MF, Folstein SE, McHugh PR (1975): “Mini-mental state." A practical method for grading the cognitive state of patients for the clinician. J Psychiatr Res 12:189-198.

Formisano E, Esposito F, Di Salle F, Goebel R (2004): Cortex-based independent component analysis of $\mathrm{fMRI}$ time series. Magn Reson Imaging 22:1493-1504.

Friedman JH (2010): Parkinson's disease psychosis 2010: a review article. Parkinsonism Relat Disord 16:553-560.

Gibb WR, Lees AJ (1988): The relevance of the Lewy body to the pathogenesis of idiopathic Parkinson's disease. J Neurol Neurosurg Psychiatr 51:745-752.

Goetz CG, Tilley BC, Shaftman SR, Stebbins GT, Fahn S, Martinez-Martin P, Poewe W, Sampaio C, Stern MB, Dodel R, Dubois B, Holloway R, Jankovic J, Kulisevsky J, Lang AE, Lees $A$, Leurgans $S$, LeWitt PA, Nyenhuis D, Olanow CW, Rascol O, Schrag A, Teresi JA, van Hilten JJ, LaPelle N, Movement Disorder Society UPDRS Revision Task Force (2008): Movement Disorder Society-sponsored revision of the Unified Parkinson's Disease Rating Scale (MDS-UPDRS): scale presentation and clinimetric testing results. Mov Disord 23:2129-2170.

Goetz CG, Vaughan CL, Goldman JG, Stebbins GT (2014): I finally see what you see: Parkinson's disease visual hallucinations captured with functional neuroimaging. Mov Disord 29:115-117.

Jardri R, Thomas P, Delmaire C, Delion P, Pins D (2013): The neurodynamic organization of modality-dependent hallucinations. Cereb Cortex 23:1108-1117.

Lee J-Y, Kim JM, Ahn J, Kim H-J, Jeon BS, Kim TW (2014): Retinal nerve fiber layer thickness and visual hallucinations in Parkinson's Disease. Mov Disord 29:61-67. 
Lefebvre S, Demeulemeester M, Leroy A, Delmaire C, Lopes R, Pins D, Thomas P, Jardri R (2016): Network dynamics during the different stages of hallucinations in schizophrenia. Hum Brain Mapp 37:2571-2586.

Lenka A, Jhunjhunwala KR, Saini J, Pal PK (2015): Structural and functional neuroimaging in patients with Parkinson's disease and visual hallucinations: A critical review.

Parkinsonism Relat Disord 21:683-691.

Leroy A, Foucher JR, Pins D, Delmaire C, Thomas P, Roser MM, Lefebvre S, Amad A, Fovet T, Jaafari N, Jardri R (2017): fMRI capture of auditory hallucinations: Validation of the twosteps method. Hum Brain Mapp 38:4966-4979.

Martinez-Martin P, Schapira AHV, Stocchi F, Sethi K, Odin P, MacPhee G, Brown RG, Naidu Y, Clayton L, Abe K, Tsuboi Y, MacMahon D, Barone P, Rabey M, Bonuccelli U, Forbes A, Breen K, Tluk S, Olanow CW, Thomas S, Rye D, Hand A, Williams AJ, Ondo W, Chaudhuri KR (2007): Prevalence of nonmotor symptoms in Parkinson's disease in an international setting; study using nonmotor symptoms questionnaire in 545 patients. Mov Disord 22:1623-1629.

Mattis S (1976): Mental status examination for organic mental syndrome in the elderly patient. In: Bellak, L, T, K, editors. Geriatric Psychiatry. New York: Grune and Stratton. pp 77-121.

Milner AD (2017): How do the two visual streams interact with each other? Exp Brain Res 235:1297-1308.

Montgomery SA, Asberg M (1979): A new depression scale designed to be sensitive to change. The British journal of psychiatry.

Muller AJ, Shine JM, Halliday GM, Lewis SJG (2014): Visual hallucinations in Parkinson's disease: theoretical models. Mov Disord 29:1591-1598.

Oldfield RC (1971): The assessment and analysis of handedness: the Edinburgh inventory. Neuropsychologia 9:97-113.

Papapetropoulos S, Katzen H, Schrag A, Singer C, Scanlon BK, Nation D, Guevara A, Levin B (2008): A questionnaire-based (UM-PDHQ) study of hallucinations in Parkinson's disease. BMC Neurol 8:21.

Pieri V, Diederich NJ, Raman R, Goetz CG (2000): Decreased color discrimination and contrast sensitivity in Parkinson's disease. J Neurol Sci 172:7-11.

Shine JM, Halliday GM, Gilat M, Matar E, Bolitho SJ, Carlos M, Naismith SL, Lewis SJG (2014): The role of dysfunctional attentional control networks in visual misperceptions in Parkinson's disease. Hum Brain Mapp 35:2206-2219.

Shine JM, Muller AJ, O'Callaghan C, Hornberger M, Halliday GM, Lewis SJ (2015): Abnormal connectivity between the default mode and the visual system underlies the manifestation of visual hallucinations in Parkinson's disease: a task-based fMRI study. NPJ Parkinsons Dis 1:15003.

Swann P, O'Brien JT (2018): Management of visual hallucinations in dementia and Parkinson's disease. Int Psychogeriatr:1-22.

Tomlinson CL, Stowe R, Patel S, Rick C, Gray R, Clarke CE (2010): Systematic review of levodopa dose equivalency reporting in Parkinson's disease. Mov Disord 25:2649-2653.

Weil RS, Schrag AE, Warren JD, Crutch SJ, Lees AJ, Morris HR (2016): Visual dysfunction in Parkinson's disease. Brain 139:2827-2843.

\section{Acknowledgment}


The authors thank Anne-Sophie Carette and Lucie Plomhause for their help in neuropsychological assessment. 

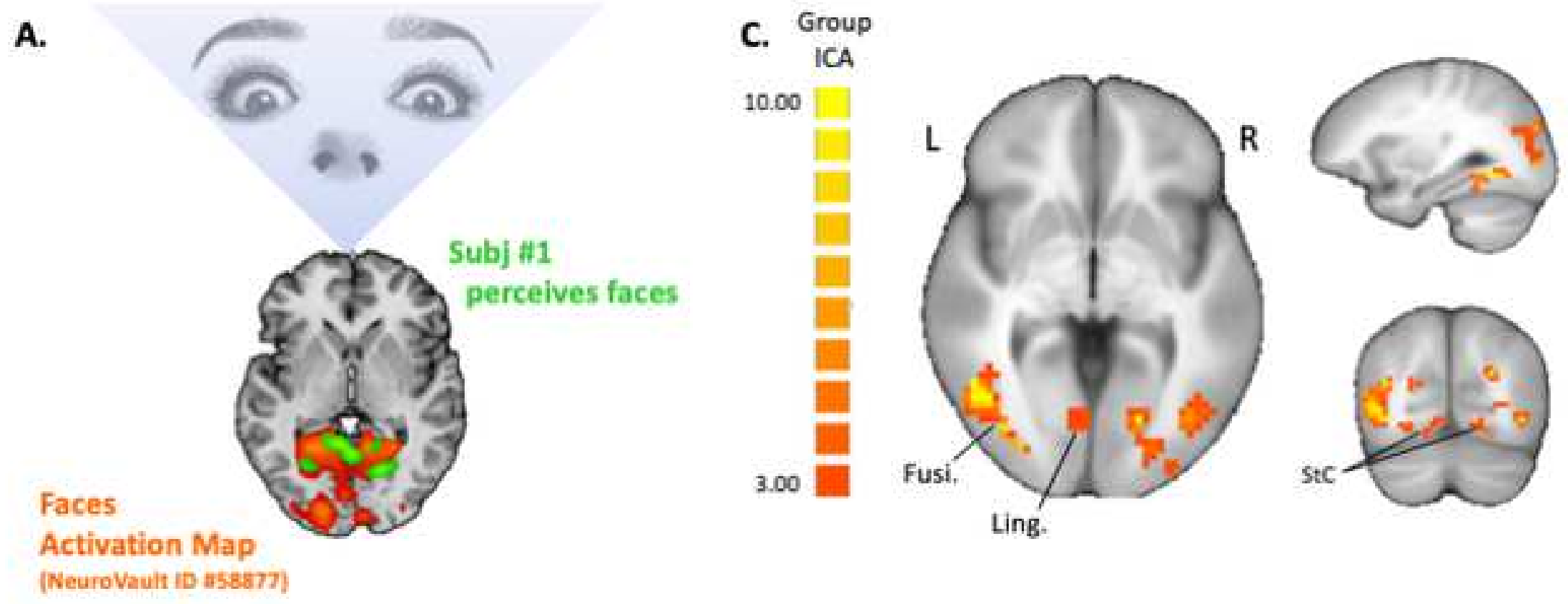

B.

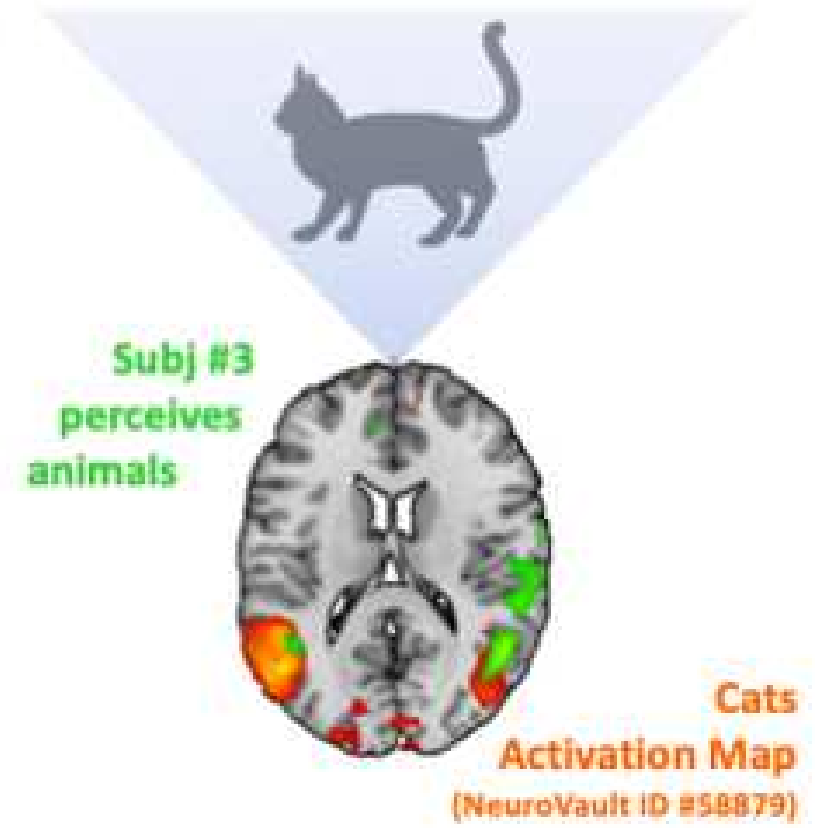

D.

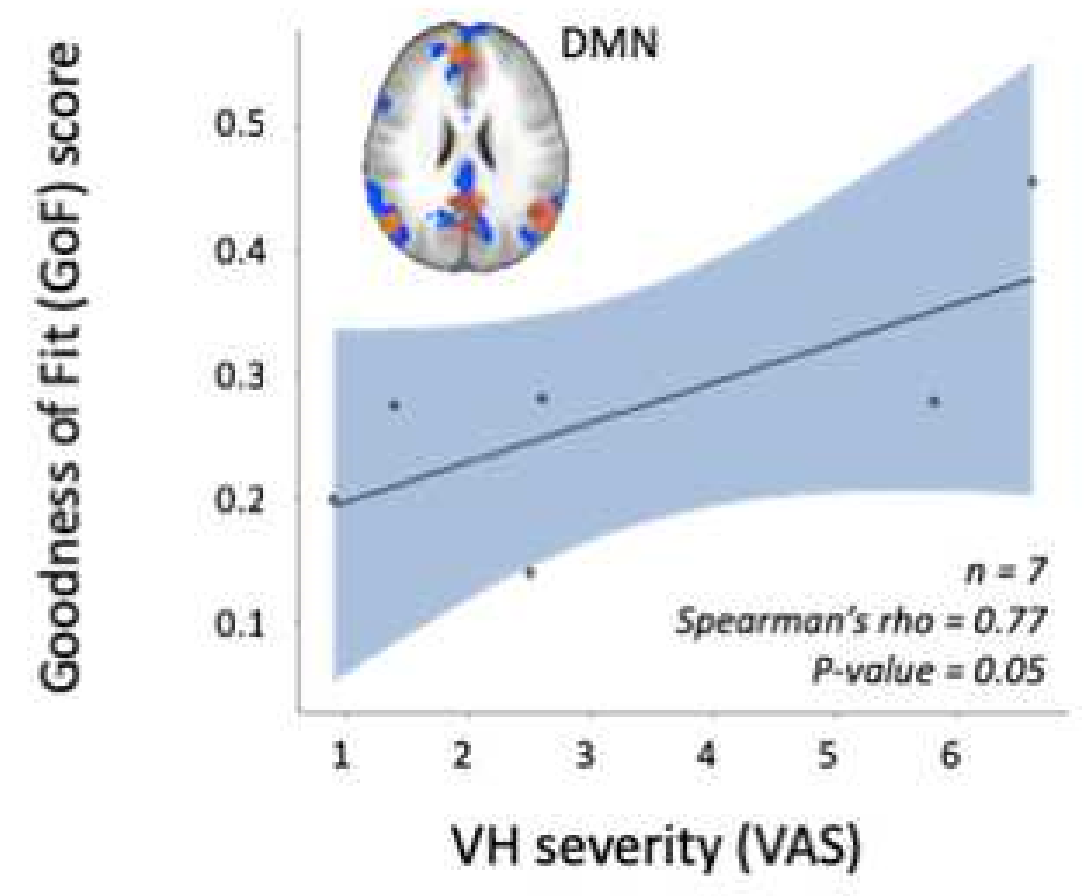


Figure 1. Functional Magnetic Resonance Imaging (fMRI) capture of visual hallucinations (VH) in patients with Parkinson Disease (PD). Cortical areas overactivated during hallucinatory experience at an individual level in (A) patient (1) who saw distorted faces and exhibited an increased signal in bilateral fusiform gyri (green) during VH, which nicely overlaps "face activation maps" from the literature (orange, NeuroVault deposit \#58877); or (B) in patient (3), who saw animal shapes and exhibited an increased signal in lateral occipital cortices (in green) during VH, which overlaps "cats activation maps" from the literature (orange, NeuroVault deposit \#58879). (C) At the group-level, ICA revealed increased connectivity in visual networks concomitant to VH, encompassing the striate cortex, and the lingual/fusiform gyri bilaterally. (D) Spatial stability of the default-mode network (DMN), as measured with a goodness-of-fit score was found positively correlated with VH severity.

Fusi: Fusiform gyrus; ICA: Independent Component Analysis; L/R: Left and Right side of the brain; Ling: Lingual gyrus; StC: Striate cortex; VAS: Visual Analogic Scale. 


\begin{tabular}{|c|c|c|c|c|c|c|c|}
\hline SUBJECTS & P01 & P03 & P05 & P09 & P04 & P06 & P08 \\
\hline HALLUCINATIONS DURING THE SCAN & Yes & Yes & Yes & Yes & No & No & No \\
\hline \multicolumn{8}{|l|}{ DEMOGRAPHIC } \\
\hline Sex & $\mathrm{F}$ & $M$ & $\mathrm{M}$ & $\mathrm{M}$ & $\mathrm{M}$ & $\mathrm{M}$ & $\mathrm{F}$ \\
\hline Formal education (y) & 14 & 8 & 10 & 8 & 8 & 11 & 8 \\
\hline \multicolumn{8}{|l|}{ CLINICAL } \\
\hline Disease duration(y) & 13 & 9 & 14 & 20 & 4 & 12 & 18 \\
\hline Hoehn \& Yahr stage & 3 & 3 & 2,5 & 3 & 2 & 3,5 & 5 \\
\hline Side of onset & Right & Right & Left & Left & Right & Right & Right \\
\hline \multicolumn{8}{|l|}{ MEDICATION } \\
\hline LEDD (mg/day) & 1125 & 1218,7 & 912,5 & 1500 & 562,5 & 800 & 1122,5 \\
\hline Agonist LEDD (mg/day) & 0 & 52 & 0 & 200 & 0 & 0 & 0 \\
\hline Cholinesterase inhibitor & Yes & No & No & No & Yes & Yes & Yes \\
\hline VH severity (0-10 VAS) & 1,4 & 2,5 & 6,8 & 0,9 & 2,6 & NA & NA \\
\hline Loss of insight & Partial & No & No & No & Partial & No & Partial \\
\hline \multicolumn{8}{|l|}{ COGNITION } \\
\hline MMSE (/30) & 19 & 25 & 24 & 25 & 19 & 23 & 21 \\
\hline Mattis DRS (/144) & 135 & 133 & 136 & 135 & 116 & 117 & 125 \\
\hline \multicolumn{8}{|l|}{ NEUROPSYCHIATRIC } \\
\hline MADRS (/60) & 6 & 8 & 6 & 18 & 10 & 15 & 12 \\
\hline PHENOMENOLOGY OF HALLUCINATIONS & NG THE SC & & & & & & \\
\hline
\end{tabular}




\begin{tabular}{|c|c|c|c|c|c|c|c|}
\hline Timing & Discontinuous & Continuous & $\begin{array}{l}\text { Several } \\
\text { occurrences } \\
\text { of more than } \\
10 \mathrm{sec} \\
\text { throughout } \\
\text { the scan }\end{array}$ & $\begin{array}{l}\text { Short } \\
\text { passages of a } \\
\text { few seconds } \\
\text { throughout } \\
\text { the } \\
\text { acquisition }\end{array}$ & NA & NA & NA \\
\hline Hallucinatory content & $\begin{array}{l}\text { Unreal people } \\
\text { and objects, } \\
\text { appearing } \\
\text { clearly, } \\
\text { motionless, } \\
\text { colored. }\end{array}$ & $\begin{array}{l}\text { Lines and } \\
\text { geometric } \\
\text { forms. Static. } \\
\text { Square with } \\
\text { black lines } \\
\text { that has } \\
\text { distorted to } \\
\text { look like a } \\
\text { black cat's } \\
\text { head during } \\
\text { the rest of } \\
\text { the } \\
\text { acquisition. }\end{array}$ & $\begin{array}{l}\text { Lights, faces, } \\
\text { shapes of } \\
\text { people or } \\
\text { animals. } \\
\text { Colored, } \\
\text { moving. } \\
\text { Sometimes } \\
\text { looking like } \\
\text { they were } \\
\text { real. }\end{array}$ & $\begin{array}{l}\text { Two people: } \\
\text { a man and a } \\
\text { woman. } \\
\text { Sometimes, } \\
\text { looking like } \\
\text { they were } \\
\text { real. Moving. }\end{array}$ & NA & NA & NA \\
\hline
\end{tabular}

Table 1. Demographic, clinical features of the patients. Phenomenology of the hallucinations is also reported for the patients with visual hallucinations during the scan.

MDS_UPDR3=Movement Disorders Society (sponsored)-Unified Parkinson's Disease Rating Scale-Part III (severity of motor symptoms); LEDD= Levodopa equivalent daily dose; UM-PDHQ= University of Miami-Parkinson's Disease Hallucination Questionnaire; $\mathrm{VH}=\mathrm{visual}$ hallucination; VAS=visual analog scale; MMSE= Mini Mental State Examination; DRS= dementia rating scale; MADRS= Montgomery and Asberg Depression Rating Scale; NA=Non Available or Not Applicable. 